\title{
Dynamic Characterization of Typical Electrical Circuits via Structural Properties
}

\author{
Sofía Avila-Becerril, Gerardo Espinosa-Pérez, and Paul Fernandez \\ Facultad de Ingeniería, UNAM, Edificio de Posgrado, Segundo Piso, Ciudad Universitaria, 04510 Ciudad de México, Mexico \\ Correspondence should be addressed to Gerardo Espinosa-Pérez; gerardoe@unam.mx
}

Received 6 March 2016; Revised 22 July 2016; Accepted 31 July 2016

Academic Editor: Carlo Cosentino

Copyright (C) 2016 Sofía Avila-Becerril et al. This is an open access article distributed under the Creative Commons Attribution License, which permits unrestricted use, distribution, and reproduction in any medium, provided the original work is properly cited.

\begin{abstract}
The characterization of a class of electrical circuits is carried out in terms of both stability properties and steady-state behavior. The main contribution is the interpretation of the electrical topology (how the elements that conform the circuits are interconnected) in terms of mathematical properties derived from the structure of their models. In this sense, at what extent the topology by itself defines the dynamic behavior of the systems is explained. The study is based on the graph theory allowing capturing, departing from the well-known Kirchhoff laws, the topology of the circuits into several matrices with specific structure. The algebraic analysis of these matrices permits identifying conditions that determine whether the system is stable in the sense of Lyapunov and the kind of steady-state behavior that it exhibits. The approach is mainly focused on typical topologies widely used in practice, namely, radial, ring, and mesh networks.
\end{abstract}

\section{Introduction}

Mathematical characterization of electrical circuits is a topic with a very long history. From the celebrated Kirchhoff laws, the maturity achieved in this field is widely recognized and reflected on classical references like $[1,2]$, among a vast number of them.

Fortunately, the richness of the available knowledge has become in an impelling force that continually opens new perspectives to take advantage of the known theory [3-5]. Indeed, modern applications, which range from microcircuits found in integrated circuit design to complex macronetworks like electrical power systems, are approached using the solid results formulated to characterize this kind of system.

One particular research activity that has received great attention is the control of electrical networks, whose interest is to modify the behavior of the circuit with the objective that some variables attain a prescribed value. In this context, several results have been reported about the reformulation of well-known features of circuits or even the establishment of new properties. Thus, it is possible to find results covering from characterization $[6,7]$ to control $[8]$ and applications that include both small [5] and large [9] size systems.
Another quite interesting approach to control electrical circuits is based on the modification of their structure to achieve a given operation. Viewing the control problem from this perspective is not new; for example, in the field of electrical power systems [10], both voltage and frequency stability problems are usually confronted by adding to the network new elements, called compensators, adequately located. This compensation action means the addition of new capacitors, inductors, and resistors in specific locations and in such a way that the desired behavior of the power system is achieved. Under the latter approach, several recent results have been reported whose interest lies in exploiting the structure of a given circuit to reach a specific goal. For example, in [11], the problem of shaping a resistive circuit behavior through the interconnection of another resistive circuit is studied, leading to a methodology denoted by "partial synthesis by interconnection" which, in turn, belongs to a more general controller design approach called Control by Interconnection (CbI) design [12]. Roughly speaking, under this perspective, the objective is to look at the controller as one dynamical system that when interconnected with other ones (the plant) generates a new dynamical system with desired properties. Considering this scenario, if it is well understood how the 
dynamic behavior of a given circuit is determined by its structure, then this knowledge can be used to redesign or to control the system in order to satisfy some prescribed specifications.

The aim of this paper is to provide an answer to the question stated above for the class of networks most used in practice, namely, radial, ring, and mesh topologies, which in the sequel will be identified as typical electrical circuits. The main objective is to recast both the stability properties and the steady-state behavior exhibited by these networks in terms of structural features of their mathematical models, in particular the matrices involved in them which are characterized by the interconnection of the different elements that compose the circuit.

From a technical perspective, the identification of the aforementioned properties is carried out using arguments from graph and matrix theory $[13,14]$ while the stability properties are formulated in terms of Lyapunov theory. Concerning the steady-state behavior, it is considered as the value to which the trajectories of the system tend as time tends to infinity; that is, these can be time-varying or constant (equilibrium points) trajectories.

In order to present the contributions of the paper, Kirchhoff's laws of a generic circuit are first formulated in terms of basic cutsets and loopsets [15]. After this, the fundamental concept of admissible trajectories that refers to the dynamic behavior that can be exhibited by the system is introduced. With this knowledge, the stability analysis of these behaviors is developed going from simple (linear) to more complex (nonlinear) structures, placing special attention on the typical networks. The last part of the paper concerns the characterization of the model components that influences the steady-state behavior of the typical topologies. A couple of illustrative case studies are included to illustrate the usefulness of the presented results. These analyses are related to the operation often found in practice given by a Mesh network equipped with Direct Current (DC) and Alternate Current (AC) voltage sources. For the former, some consensus operation [16] is concluded, while for the latter it is evidenced that compensation procedures can be developed in a systematic way.

The rest of the paper is organized as follows. In Section 2, using well-known results from the graph theory, Kirchhoff's laws are stated, in terms of basic cutsets and loopsets, for a model that includes a broad class of electrical circuits, while Section 3 is devoted to the presentation of its dynamic model. In Section 4, the stability analysis is carried out and the bases for the characterization of the steady-state behavior of typical networks are included in Section 5. The usefulness of the presented results is illustrated in Section 6. Some concluding remarks are discussed in Section 7.

\section{Electrical Circuit Graphs}

An electrical network can be defined as an oriented graph $G$ consisting of a finite set of nodes $\mathscr{V}(G)=\left\{v_{1}, v_{2}, \ldots, v_{n}\right\}$ and a finite set of edges $\mathscr{E}(G)=\left\{e_{1}, e_{2}, \ldots, e_{b}\right\}$ such that $\mathscr{E}$ is a subset of $\mathscr{V}^{2}$, with pairs of $\mathscr{V}$ where no self-loops are allowed.
In this case, the set of $n$ nodes is the interconnection points of elements whereas the set of $b$ edges is associated, one to one, with lumped one-port (two-terminal) elements. Additionally, it is considered that the graph is connected in the sense that each node can be reached from any other node by tracing a path through the edges.

With each edge (each lumped one-port element), a voltage $v$ across its terminals and a current $i$ that flows through it are associated. The orientation of the edge coincides with the direction of a positive current and a decreasing voltage.

Once the lumped elements are interconnected, their port variables must satisfy the constraints stated by the Kirchhoff Current and Voltage Laws (KCL and KVL, resp.) that in this paper are stated in terms of basic cutsets and basic loopsets for a given spanning tree $[14,15]$. A basic cutset (or fundamental cutset) is composed of one edge of the tree (branch) and some or all of the edges of the cotree (chords). A basic loopset (or fundamental cycle) is conformed by one chord and some or all of the branches in such a way that a closed loop is formed. In this way, there are two natural matrices associated with the graph, the basic cutset matrix $C_{b} \in \mathbb{R}^{(n-1) \times b}$ and the basic loopset matrix $B_{b} \in \mathbb{R}^{(b-n+1) \times b}$, so that KCL and KVL are given, respectively, by

$$
\begin{aligned}
& C_{b} i=0 ; \\
& B_{b} v=0 .
\end{aligned}
$$

If the currents $i$ and voltages $v$ associated with the $b$ edges are ordered in such a way that

$$
\begin{aligned}
& i=\left[\begin{array}{l}
i_{t} \\
i_{c}
\end{array}\right] \in C_{1} ; \\
& v=\left[\begin{array}{l}
v_{t} \\
v_{c}
\end{array}\right] \in C^{1},
\end{aligned}
$$

with $i_{t} \in \mathbb{R}^{(n-1)}$ and $v_{t} \in \mathbb{R}^{(n-1)}$ being the currents and voltages of the tree and $i_{c} \in \mathbb{R}^{(b-n+1)}$ and $v_{c} \in \mathbb{R}^{(b-n+1)}$ being the currents and voltages of the cotree, respectively, it is possible to write down the network constraints [14] as

$$
\begin{aligned}
& i_{t}=-H i_{c}, \\
& v_{c}=H^{T} v_{t},
\end{aligned}
$$

where $H \in \mathbb{R}^{(n-1) \times(b-n+1)}$ is called the fundamental loop matrix.

From this last expression, it can be noticed that the $i$ th row of $H$ indicates which of the cotree elements is incident to the $i$ th basic cutset and that, in correspondence, the $j$ th column shows which of the tree elements belongs to the $j$ th basic loopset.

At this point, it is important to state that the matrix $H$ determines the topological structure of the system and its dynamic behavior. The analysis of this matrix is the main interest of the paper. All the results presented throughout this contribution are related to its structure. 


\section{Electrical Circuit Dynamic}

In this section, the dynamic equations for the electrical circuit presented in Section 2 are obtained following ideas of, for example, [11]. The analysis is restricted to networks where capacitor-only loops and inductor-only cutsets are not admitted [1]. In addition, the circuit elements are grouped such that voltage sources, all the capacitors, and some resistors appear at the tree, while inductors and the rest of the resistors are in the cotree (For the sake of simplicity, presentation of the current sources will be omitted from the analysis.), leading to

$$
\begin{aligned}
& i_{t}=\left[\begin{array}{l}
i_{1} \\
i_{C} \\
i_{R t}
\end{array}\right] ; \\
& v_{c}=\left[\begin{array}{c}
v_{R c} \\
v_{L}
\end{array}\right],
\end{aligned}
$$

with voltage tree and current cotree vectors

$$
\begin{aligned}
& v_{t}=\left[\begin{array}{c}
v_{1} \\
v_{C} \\
v_{R t}
\end{array}\right] ; \\
& i_{c}=\left[\begin{array}{c}
i_{R c} \\
i_{L}
\end{array}\right],
\end{aligned}
$$

where $v_{1}, i_{1} \in \mathbb{R}^{n_{1}}, v_{C}, i_{C} \in \mathbb{R}^{n_{2}}, v_{R t}, i_{R t} \in \mathbb{R}^{n_{3}}$, such that $n_{1}+n_{2}+n_{3}=n-1$, and $v_{R c}, i_{R c} \in \mathbb{R}^{n_{4}}, v_{L}, i_{L} \in \mathbb{R}^{n_{5}}$, with $n_{4}+n_{5}=b-(n-1)$.

If the total stored energy of the circuit $H_{a}: \mathbb{R}^{n_{2} \times n_{5}} \rightarrow$ $\mathbb{R}_{\geq 0}$ is defined as $H_{a}(q, \phi)=V_{q}(q)+V_{\phi}(\phi)$, it is well known [15] that the port variables of the capacitors, inductors, and resistors can be represented as

$$
\begin{aligned}
\dot{q} & =i_{C}, \\
v_{C} & =\frac{\partial H_{a}(q, \phi)}{\partial q}=\nabla_{q} H_{a} \\
\dot{\phi} & =v_{L}, \\
i_{L} & =\frac{\partial H_{a}(i, \phi)}{\partial \phi}=\nabla_{\phi} H_{a} \\
i_{R t} & =-f_{t}\left(v_{R t}\right), \\
v_{R c} & =-f_{c}\left(i_{R c}\right),
\end{aligned}
$$

where $f_{t}$ and $f_{c}$ are assumed to be bijective functions.

The partition introduced above results in the fact that matrix $H$, in turn, can be partitioned as

$$
H=\left[\begin{array}{ll}
H_{1 R} & H_{1 L} \\
H_{C R} & H_{C L} \\
H_{R R} & H_{R L}
\end{array}\right],
$$

where the subscript stands for the interconnections between tree and cotree elements.
Remark 1. The partition of matrix $H$ presented in (7) is important from the point of view of the analysis developed in this paper. It allows, as will be clear below, identifying which part of the model influences the stability properties of the system and which part determines the steady-state behavior.

As already reported in the literature, substitution of (6a)(6c) into (3) leads to the dynamical model given by

$$
\dot{x}=\mathbb{J} \nabla_{x} H_{a}(x)+\mathbb{F}\left(x, v_{1}, v_{R t}, i_{R c}\right)+\mathbb{G} E_{1}
$$

under the definitions

$$
\begin{aligned}
x & =\left[\begin{array}{l}
q \\
\phi
\end{array}\right] ; \\
\nabla_{x} H_{a}(x) & =\left[\begin{array}{l}
\nabla_{q} H_{a}(x) \\
\nabla_{\phi} H_{a}(x)
\end{array}\right] ; \\
E_{1} & =\left[\begin{array}{l}
v_{1} \\
0
\end{array}\right],
\end{aligned}
$$

with matrices

$$
\begin{aligned}
\mathbb{V} & =\left[\begin{array}{cc}
0 & -H_{C L} \\
H_{C L}^{T} & 0
\end{array}\right] ; \\
\mathbb{G} & =\left[\begin{array}{cc}
0 & 0 \\
H_{1 L}^{T} & 0
\end{array}\right] \\
\mathbb{F}\left(x, v_{1}, v_{R t}, i_{R c}\right) & =\left[\begin{array}{cc}
0 & -H_{C R} \\
H_{R L}^{T} & 0
\end{array}\right]\left[\begin{array}{l}
v_{R t} \\
i_{R c}
\end{array}\right],
\end{aligned}
$$

where

$$
\left[\begin{array}{c}
v_{R t} \\
i_{R c}
\end{array}\right]=\left[\begin{array}{c}
-f_{t}^{-1}\left(-H_{R R} i_{R c}-H_{R L} \nabla_{\phi} H_{a}(x)\right) \\
-f_{c}^{-1}\left(H_{1 R}^{T} v_{1}+H_{R R}^{T} v_{R t}+H_{C R}^{T} \nabla_{q} H_{a}(x)\right)
\end{array}\right],
$$

and complemented by the algebraic constraint

$$
i_{1}=-H_{1 R} i_{R c}-H_{1 L} i_{L}
$$

It is important to notice that if we concentrate on circuits with linear resistive elements, then

$$
\begin{gathered}
i_{R t}=-R_{t}^{-1} v_{R t}, \\
v_{R c}=-R_{c} i_{R c},
\end{gathered}
$$

where $R_{t}=R_{t}^{T}>0$, a diagonal matrix with entries in the resistances of the tree resistors, and $R_{c}=R_{c}^{T}>0$ also diagonal and composed of the resistances of the cotree. As usual, all 
circuit resistances are assumed to be strictly positive [11]. For this linear representation, it holds that

$$
\begin{aligned}
& {\left[\begin{array}{c}
v_{R t} \\
i_{R c}
\end{array}\right]=\left[\begin{array}{cc}
I_{2} & R_{t} H_{R R} \\
-R_{c}^{-1} H_{R R}^{T} & I_{3}
\end{array}\right]^{-1}} \\
& \quad \times\left\{\left[\begin{array}{cc}
0 & -R_{t} H_{R L} \\
R_{c}^{-1} H_{C R}^{T} & 0
\end{array}\right] \nabla_{x} H_{a}(x)\right. \\
& \left.+\left[\begin{array}{c}
0 \\
R_{c}^{-1} H_{1 R}^{T} v_{1}
\end{array}\right]\right\},
\end{aligned}
$$

where $I_{2} \in \mathbb{R}^{n_{3} \times n_{3}}$ and $I_{3} \in \mathbb{R}^{n_{4} \times n_{4}}$ are identities matrices and, consequently, models (8) and (15) can be equivalently represented as a port-controlled Hamiltonian system with dissipation [17] of the form

$$
\dot{x}=[J-R] \nabla_{x} H_{a}(x)+G E_{1}
$$

defining

$$
\begin{aligned}
& J=\left[\begin{array}{cc}
0 & J_{12} \\
-J_{12}^{T} & 0
\end{array}\right] ; \\
& R=\left[\begin{array}{cc}
R_{1} & 0 \\
0 & R_{2}
\end{array}\right] \\
& G \\
& =\left[\begin{array}{cc}
-H_{C R}\left[R_{c}+H_{R R}^{T} R_{t} H_{R R}\right]^{-1} H_{1 R}^{T} & 0 \\
H_{1 L}^{T}-H_{R L}^{T} R_{t} H_{R R}\left[R_{c}+H_{R R}^{T} R_{t} H_{R R}\right]^{-1} H_{1 R}^{T} & 0
\end{array}\right],
\end{aligned}
$$

where $J=-J^{T}, R=R^{T} \geq 0$, and

$$
\begin{aligned}
& J_{12}=H_{C R}\left[R_{c}+H_{R R}^{T} R_{t} H_{R R}\right]^{-1} H_{R R}^{T} R_{t} H_{R L}-H_{C L} \\
& R_{1}=H_{C R}\left[R_{c}+H_{R R}^{T} R_{t} H_{R R}\right]^{-1} H_{C R}^{T} \\
& R_{2}=H_{R L}^{T}\left[R_{t}^{-1}+H_{R R} R_{c}^{-1} H_{R R}^{T}\right]^{-1} H_{R L}
\end{aligned}
$$
order.

The following remarks about the obtained model are in

Remark 2. The algebraic constraint (13) represents the current demanded to the voltage sources for a given operation point. Along the paper, it is considered that the sources are ideal; that is, they can supply any amount of current, and therefore the constraint is always satisfied.

Remark 3. Notice that the matrix $H_{C L}$, which includes the relationship between capacitors and inductances, appears in a skew-symmetric form in the model. It will be shown that this condition implies that it does not modify the energy balance equation of the circuit. However, it will be fundamental to determine the steady-state behavior of the circuit.
Remark 4. Also related to matrix $J$, the time derivative of $H_{a}(x)$ along the trajectories of (16), considering $E_{1}=0$, is given by

$$
\dot{H}_{a}(x)=\left(\nabla_{x} H_{a}(x)\right)^{T} \mathbb{F}\left(x, v_{1}, v_{R t}, i_{R c}\right)
$$

putting in evidence the fact that stability of the network depends on the matrices $H_{C R}, H_{R L}, H_{R R}$, and $H_{1 R}$.

Remark 5. If the network is conformed by linear elements, the property above is stronger since

$$
\dot{H}_{a}(x)=\left(\nabla_{x} H_{a}(x)\right)^{T} R \nabla_{x} H_{a}(x) \leq 0 .
$$

Thus, since $R=R^{T}>0$, a solution $x^{\star}$ of $y=\nabla_{x}^{\star} H_{a}\left(x^{\star}\right)=$ 0 will be asymptotically stable if the system is zero state detectable considering as output the variable $y$ and $x^{\star}$ is a minimum argument of the scalar function $H_{a}(x)$.

\section{Structural Properties for Stability}

The purpose of this section is to identify conditions for stability when $E_{1}$ is different from zero. In this context, it is necessary to identify the steady-state trajectories that are achievable by the system, denoted by admissible trajectories. These behaviors are solution of

$$
\dot{x}^{\star}=\mathbb{J} \nabla_{x^{\star}} H_{a}\left(x^{\star}\right)+\mathbb{F}\left(x^{\star}, v_{1}^{\star}, v_{R t}^{\star}, i_{R c}^{\star}\right)+\mathbb{G} E_{1}^{\star},
$$

where the existence of an input $v_{1}^{\star}$ that generates the behavior $x^{\star}$ has been implicitly assumed. A particular case is when $x^{\star}$ is an equilibrium point. Under this condition, $v_{1}^{\star}$ is constant and the steady-state operation (equilibrium point) is input dependent and is determined by

$$
\mathbb{J} \nabla_{x^{\star}} H_{a}\left(x^{\star}\right)+\mathbb{F}\left(x^{\star}, v_{1}^{\star}, v_{R t}^{\star}, i_{R c}^{\star}\right)+\mathbb{G} E_{1}^{\star}=0 .
$$

The admissible trajectories directly depend on the matrix $H$ presented in (7). Therefore, the results presented below concentrate the attention in finding structural properties of this matrix such that some stability properties are guaranteed.

The first result states the stronger result concerning the achievable admissible trajectories although the simplest structure for the network, namely, conditions for $H$, is identified to guarantee tracking of a time-varying solution of (21) but this result applies only when the circuit is composed of elements that exhibit a linear constitutive relationship, that is, when the total stored energy $H: \mathbb{R}^{n_{2}} \times \mathbb{R}^{n_{5}} \rightarrow \mathbb{R}_{>0}$ takes the form

$$
H_{a}(x)=\frac{1}{2} x^{T} P x ; \quad P=\operatorname{diag}\left\{C^{-1}, L^{-1}\right\}=P^{T}>0
$$

with positive and diagonal matrices $L \in \mathbb{R}^{n_{5} \times n_{5}}$ and $C \in$ $\mathbb{R}^{n_{2} \times n_{2}}$ of inductances and capacitances, respectively. In this case, it is clear that

$$
\nabla_{x} H_{a}(x)=P x
$$

while the resistors satisfy (14). 
Proposition 6. Consider a linear electrical network described by (16) and (23) with $v_{1}(t)$ being a time-varying input such that its steady-state behavior, characterized by

$$
\dot{x}^{\star}=[J-R] P x^{\star}+G E_{1}^{\star},
$$

is well posed.

Under these conditions,

$$
\lim _{t \rightarrow \infty} \tilde{x}=0
$$

with $\tilde{x}=x-x^{\star}$ if

$$
\operatorname{ker}\left\{H_{C R}^{T}\right\}=\operatorname{ker}\left\{H_{R L}\right\}=0 \text {. }
$$

Proof. Under the linear characteristic assumed for the system, it is possible to describe the error dynamic by

$$
\dot{\tilde{x}}=[J-R] P \widetilde{x}+G \widetilde{E}_{1}
$$

with the energy-like function

$$
H_{a}(\tilde{x})=\frac{1}{2} \tilde{x}^{T} P \tilde{x}
$$

and the identities $\widetilde{v}_{C}=\nabla_{\widetilde{x}_{1}} H_{a}(\widetilde{x})=C^{-1} \widetilde{q}, \widetilde{i}_{L}=\nabla_{\widetilde{x}_{2}} H_{a}(\widetilde{x})=$ $L^{-1} \tilde{\phi}$.

Since the purpose is to analyze the stability properties of the equilibrium point $\left(\widetilde{x}, \widetilde{v}_{1}\right)=(0,0)$ of $(28)$, function (29) can be considered as Lyapunov function candidate. Hence, it is straightforward to show that its time derivative along the trajectories of (28) satisfies

$$
\dot{H}_{a}=-\tilde{x}^{T} P R P \widetilde{x} \leq 0
$$

which can be written in an equivalent way as

$$
\dot{H}_{a}=-z R_{T}^{-1} z
$$

with $R_{T}=\operatorname{diag}\left\{R_{11}, R_{22}\right\}$ and $R_{11}, R_{22}$ being symmetric positive definite matrices defined by

$$
\begin{aligned}
& R_{11}=R_{c}+H_{R R}^{T} R_{t} H_{R R} \\
& R_{22}=R_{t}^{-1}+H_{R R} R_{c}^{-1} H_{R R}^{T}
\end{aligned}
$$

while

$$
z=\left[\begin{array}{c}
H_{C R}^{T} \widetilde{v}_{C} \\
H_{R L} \widetilde{i}_{L}
\end{array}\right] .
$$

The proof is completed by noting that the maximal invariant set where $\dot{\vec{H}}_{a}=0$ is $z=0$, which leads to $H_{C R}^{T} \widetilde{v}_{C}=0$ and $H_{R L} \widetilde{i}_{L}=0$. Therefore, the necessity to guarantee that $\widetilde{v}_{C}=\widetilde{i}_{L}=0$ are the only solutions that satisfy these constraints brings out $\operatorname{ker}\left\{H_{C R}^{T}\right\}=\operatorname{ker}\left\{H_{R L}\right\}=0$ as the sufficient conditions that assure asymptotic stability of $\left(\widetilde{x}, \widetilde{v}_{1}\right)=(0,0)$.
Remark 7. An interesting feature in the proof of the presented result is that the zero state detectability that could be invoked in (31) in order to conclude asymptotic stability has been recast, via (33), in terms of the structural properties of the system. In this scenario, to what extent the stability properties of the network are determined by its structure has been stated.

Remark 8. Even though Proposition 6 is related to linear systems, in the authors' opinion, it is important since this kind of representation for electrical networks is still widely used under a great variety of applications.

In the proposition below, the result presented above is extended in the sense that the asymptotic stability properties are still guaranteed considering nonlinear capacitors and inductors. However, this is achieved at the expense that the admissible trajectories are no longer time-varying but equilibrium points.

Proposition 9. Consider an electrical network described by model (8) with $v_{1}^{\star}$ being a constant input such that the steadystate behavior is well posed. In addition, assume the following:

(A.1) The resistors involved in the circuit are characterized by linear constitutive relationships satisfying (15).

(A.2) The equilibrium point $x^{\star}$ that corresponds to $v_{1}^{\star}$ locally satisfies $x^{\star}=\operatorname{argmin}\left\{H_{a}(x)\right\}$.

Under these conditions, the equilibrium point $\left(x^{\star}, v_{1}^{\star}\right)$ is locally asymptotically stable if

$$
\operatorname{ker}\left\{H_{C R}^{T}\right\}=\operatorname{ker}\left\{H_{R L}\right\}=0 \text {. }
$$

Proof. In this case, the total stored energy $H_{a}(x)$ is a nonlinear function. Therefore, under assumption (A.1), the dynamic behavior of the system is described by (16) while its equilibria are characterized by the solutions of

$$
[J-R] \nabla_{x^{\star}} H_{a}\left(x^{\star}\right)+G E_{1}^{\star}=0 .
$$

If assumption (A.2) holds, then, following ideas reported in [18], it is possible to consider the Lyapunov function candidate $H_{0}: \mathbb{R}^{n_{2}+n_{5}} \rightarrow \mathbb{R}_{\geq 0}$ given by

$$
\begin{aligned}
H_{0}(x)= & H_{a}(x)-x^{T} \nabla_{x^{\star}} H_{a}\left(x^{\star}\right) \\
& -\left(H_{a}\left(x^{\star}\right)-x^{\star T} \nabla_{x^{\star}} H_{a}\left(x^{\star}\right)\right)
\end{aligned}
$$

whose time derivative along the trajectories of (16), under the condition $v_{1}=v_{1}^{\star}$, yields

$$
\begin{aligned}
\dot{H}_{0}(x)= & -\left(v_{C}-v_{C}^{\star}\right)^{T} R_{1}\left(v_{C}-v_{C}^{\star}\right) \\
& -\left(i_{L}-i_{L}^{\star}\right)^{T} R_{2}\left(i_{L}-i_{L}^{\star}\right)
\end{aligned}
$$

with $R_{1}, R_{2}$ defined in (17a), where we exploited the fact that, in spite of the nonlinear nature of $H_{a}(x)$, the identities introduced in (6a) and (6b) are valid.

The proof is completed following a similar procedure to Proposition 6 since it is possible to get that

$$
\dot{H}_{0}(x)=-z^{T} R_{T}^{-1} z
$$


with

$$
z=\left[\begin{array}{c}
H_{C R}^{T} \widetilde{v}_{C} \\
H_{R L} \widetilde{i}_{L}
\end{array}\right]
$$

Remark 10. Notice that the function $H_{0}(x)$ qualifies as Lyapunov function candidate only for equilibrium points. Current research is carried out trying to find an alternative analysis to deal with the tracking problem but, to the authors' knowledge, this imposes an open problem in the related literature.

Remark 11. If $x^{\star}$ is a global minimum of $H_{a}(x)$, with $H_{a}(x)$ radially unbounded, then the equilibrium point is globally asymptotically stable.

Motivated by the identified role played by matrices $H_{C R}$ and $H_{R L}$ in the presented results, the next two propositions are focused on a practically important class of circuits which is characterized by a particular interconnection, first among resistors and inductors and second among resistors and capacitors.

Property 1. If the number of tree resistors is equal to the number of inductors and they are one-to-one series connected, then

$$
\begin{aligned}
& H_{R L}=I_{2} \in \mathbb{R}^{n_{3} \times n_{3}} ; \\
& H_{R R}=0_{1} \in \mathbb{R}^{n_{3} \times n_{4}},
\end{aligned}
$$

with $I_{2}$ as already defined and $0_{1}$ being a zero matrix.

Proof. If there exists the same number of tree resistors as inductors, then $n_{3}=n_{5}$. On the other hand, from (3), the constraint

$$
i_{R t}=H_{R R} i_{R c}+H_{R L} i_{L}
$$

must be satisfied. Hence, the fact that tree resistor currents $i_{R t}$ must be, one-to-one, equal to the inductor currents $i_{L}$ leads to the results stated in (40).

Property 2. If the number of cotree resistors is equal to the number of capacitors and they are one-to-one parallel connected, then

$$
\begin{aligned}
& H_{C R}=I_{3} \in \mathbb{R}^{n_{2} \times n_{2}} ; \\
& H_{1 R}=0_{2} \in \mathbb{R}^{n_{1} \times n_{2}},
\end{aligned}
$$

with $I_{3}$ as already defined and $0_{2}$ being a zero matrix.

Proof. If there exists the same number of cotree resistors as capacitors, then $n_{2}=n_{4}$. In this case, also obtained from (3), the constraint that must be satisfied is

$$
v_{R c}=H_{1 R}^{T} v_{1}+H_{C R}^{T} v_{C}+H_{R R}^{T} v_{R t},
$$

where, due to Property $1, H_{R R}=0_{1}$, leading to the expression

$$
v_{R c}=H_{1 R}^{T} v_{1}+H_{C R}^{T} v_{C} .
$$

The proof concludes by noting that each entry of $v_{R c}$ must be equal to its corresponding entry in $v_{C}$, a condition that holds only if (42) is true.

Remark 12. An immediate implication of the presented properties is that the networks that enjoy them automatically satisfy the conditions stated in Propositions 6 and 9 to guarantee asymptotic stability of the admissible trajectories.

Remark 13. In the context of this paper, the stated properties are important since all the considered typical networks enjoy them, as will be shown in Section 5 .

In order to present the last result of this section, writing model (8) under the effect of Properties 1 and 2 is convenient. Hence, the dynamical behavior of the electrical network is described by

$$
\dot{x}=\mathbb{J} \nabla_{x} H_{a}(x)+\mathbb{F}_{1}(x)+\mathbb{G} E_{1},
$$

where

$$
\mathbb{F}_{1}(x)=\left[\begin{array}{c}
-f_{c}^{-1}\left(\nabla_{q} H_{a}(x)\right) \\
-f_{t}^{-1}\left(\nabla_{\phi} H_{a}(x)\right)
\end{array}\right] .
$$

Due to the achieved drastic simplification in the model structure, which illustrates how a proper interconnection of the elements determines to a large extent the dynamic behavior of the system, previous results can be extended by relaxing the assumptions about $f_{c}(\cdot)$ and $f_{t}(\cdot)$, which under the new conditions only depend on the system state.

Proposition 14. Consider the electrical network described by (45) with $v_{1}^{\star}$ being a constant input such that the steady-state behavior is well posed. Assume (A.2) holds and in addition assume the following:

(A.3) The maps $f_{c}(\cdot)$ and $f_{t}(\cdot)$ define incremental output strictly passive operators in the sense that

$$
\begin{aligned}
& \left(x_{1}-x_{2}\right)^{T}\left[f_{c}^{-1}\left(x_{1}\right)-f_{c}^{-1}\left(x_{2}\right)\right]>0 \\
& \left(x_{1}-x_{2}\right)^{T}\left[f_{t}^{-1}\left(x_{1}\right)-f_{t}^{-1}\left(x_{2}\right)\right]>0
\end{aligned}
$$

hold for $x_{1} \neq x_{2}$ and considering $x_{i}$ as input.

Under these conditions, the equilibrium point $\left(x^{\star}, v_{1}^{\star}\right)$ is locally asymptotically stable.

Proof. The equilibria of the system are characterized by

$$
\mathbb{J} \nabla_{x^{\star}} H_{a}\left(x^{\star}\right)+\mathbb{F}_{1}\left(x^{\star}\right)+\mathbb{G} E_{1}^{\star}=0 .
$$

Thus, if (A.2) holds, it is possible to consider the Lyapunov function candidate $H_{0}: \mathbb{R}^{n_{2}+n_{5}} \rightarrow \mathbb{R}_{\geq 0}$, reported in 
[18] and defined in (36), which leads, under the condition $v_{1}=v_{1}^{\star}$, to

$$
\begin{aligned}
& \dot{H}_{0}(x) \\
& \quad=-\left(\nabla_{x} H_{a}(x)-\nabla_{x^{\star}} H_{a}\left(x^{\star}\right)\right)^{T}\left[\mathbb{F}_{1}(x)-\mathbb{F}_{1}\left(x^{\star}\right)\right] .
\end{aligned}
$$

Using identities (6a) and (6b), this expression can be equivalently written as

$$
\begin{aligned}
\dot{H}_{0}(x)= & -\left(v_{C}-v_{C}^{\star}\right)^{T}\left[f_{c}^{-1}\left(v_{C}\right)-f_{c}^{-1}\left(v_{C}^{\star}\right)\right] \\
& -\left(i_{L}-i_{L}^{\star}\right)^{T}\left[f_{t}^{-1}\left(i_{L}\right)-f_{t}^{-1}\left(i_{L}^{\star}\right)\right] .
\end{aligned}
$$

Since (A.3) holds, it is clear that $\dot{H}_{0}(x)<0$ with maximal invariance set defined as

$$
\varepsilon=\left\{(q, \phi) \mid\left(v_{C}-v_{C}^{\star}\right)=0,\left(i_{L}-i_{L}^{\star}\right)=0\right\}
$$

which concludes the proof.

Remark 15. It must be clear that if the dissipation functions $f_{c}(\cdot)$ and $f_{t}(\cdot)$ define linear mappings, introduction of Properties 1 and 2 does not destroy the results presented either in Proposition 6 or in Proposition 9.

\section{Topological Structure of Typical Networks}

In this section, the dynamic characterization started in Section 4 is complemented for the class of typical electrical networks. The main objective of the analysis is to explicitly state the structure of the matrix $H$ for the aforementioned typical networks. In this sense, the usefulness of this study is twofold:

(i) With this structure at hand, it is possible to determine in a systematic way the stability properties and steadystate behavior of the networks.

(ii) An interpretation is provided, given via structural characterization, to the widely recognized advantages exhibited by these networks, for example, reliability properties.

In order to present the results mentioned above, first a generic network that captures in a unified way the characteristics of the three typical networks is considered. Once the topology of this network is identified, each particular case is presented.

The first condition that the model of the generic network satisfies is that Properties 1 and 2 stated in Section 4 hold. Hence, its dynamic behavior is represented by model (45) leaving $H_{C L} \in \mathbb{R}^{n_{2} \times n_{5}}$ and $H_{1 L} \in \mathbb{R}^{n_{1} \times n_{5}}$ as the two matrices that remain to be characterized; that is, it is necessary to consider the interconnections between capacitors and inductors, for the former matrix, and sources and inductors, for the latter.

Consider that the $n_{5}$ inductors are divided into three types, namely, $n_{r} r$-inductors that belong to a trajectory that connects a source with a capacitor, $n_{s} s$-inductors that belong to a trajectory that connects a source with another source, and $n_{p} p$-inductors that belong to a trajectory that connects a capacitor with another capacitor, such that $n_{r}+n_{s}+n_{p}=n_{5}$. Moreover, assume that the capacitors belong at least to one of the following classes:

(C.1) The ith capacitor, $i \in\left\{1, \ldots, n_{2}\right\}$, shares cutset with $r_{i} r$-inductors.

(C.2) The $i$ th capacitor, $i \in\left\{1, \ldots, n_{2}\right\}$, shares cutset with $p_{i} p$-inductors.

However, the voltages source satisfies the following:

(C.3) The $i$ th voltage source, $i \in\left\{1, \ldots, n_{1}\right\}$, shares cutset with $m_{i} \in\left\{1, \ldots, n_{5}\right\}$ inductors.

Under the above classification, the variables associated with capacitors and inductors can be organized such that the rows of matrix $H_{C L}$ can be divided into two parts: the first corresponding to capacitors that hold simultaneously conditions (C.1) and (C.2) and the second corresponding to those capacitors that hold only with condition (C.2). As will be clear below, capacitors that satisfy only condition (C.1) are included as a particular case of the former.

On the other hand, the columns of $H_{C L}$ are divided into three blocks each one corresponding to $r-, s^{-}$, and $p^{-}$ inductors, respectively. Moreover, the $r_{i}$ inductors connected to the $i$ th capacitor as in (C.1) are grouped for each of the capacitors connected to them. form

Following the stated organization, matrix $H_{C L}$ takes the

$$
-H_{C L}=\left[\begin{array}{cccccc}
\mathbf{1}_{r_{1}}^{T} & 0 & \cdots & 0 & 0_{s} & N_{1} \\
0 & \mathbf{1}_{r_{2}}^{T} & \cdots & 0 & 0_{s} & N_{2} \\
\vdots & \vdots & \ddots & \vdots & \vdots & \vdots \\
0 & 0 & \cdots & \mathbf{1}_{r_{z}}^{T} & 0_{s} & N_{z} \\
0 & 0 & \cdots & 0 & 0_{s} & N_{z+1} \\
\vdots & \vdots & \ddots & \vdots & \vdots & \vdots \\
0 & 0 & \cdots & 0 & 0_{s} & N_{n_{2}}
\end{array}\right],
$$

where one has the following:

(i) The minus sign appears since, from (3), the constraint that must be satisfied is

$$
i_{C}=-H_{C L} i_{L}
$$

considering that $H_{C R}=0$.

(ii) $\mathbf{1}_{r_{i}}^{T} \in \mathbb{R}^{1 \times r_{i}}, i=1, \ldots, z$, are vectors filled with ones denoting the condition stated in (C.1). In this case, it has been assumed that there exist $z$ capacitors of this kind. In addition, it holds that $r_{1}+r_{2}+\cdots+r_{z}=n_{r}$.

(iii) The zero columns $0_{s} \in \mathbb{R}^{1 \times n_{s}}$ reflect the fact that any capacitor can be connected to $s$ type inductors.

(iv) Row vectors $N_{i} \in \mathbb{R}^{1 \times n_{p}}$ include the possibility that a given capacitor can be simultaneously connected 
to $r$ and $p$ type inductors. If the $i$ th capacitor is connected to one of the $p$-inductors, a 1 appears in the corresponding entry; otherwise, a 0 appears. If the capacitor only holds with (C.1), the $N_{i}$ vector is the zero vector.

(v) In the rows that go from $z+1$ to $n_{2}$, only vectors $N_{i}$ appear since they correspond to (C.2) class capacitors.

Two consequences of structure (52) are presented below.

Property 3. Each column of the matrix $N=\operatorname{col}\left\{N_{1}\right.$, $\left.\ldots, N_{n_{2}}\right\} \in \mathbb{R}^{n_{2} \times n_{p}}$ is composed of one 1 and one -1 and the rest of the entries equal to zero.

Proof. The columns of matrix $N$ denote how the capacitors are connected to other capacitors. Since for each pair of capacitors this connection is carried out by one $p$-inductor, the current leaves one cutset and enters another, which is represented by the 1 in one row and the -1 in the other row.

The second property is actually a corollary of Property 3.

Property 4. The vector $\mathbf{1}_{n_{2}} \in \mathbb{R}^{n_{2}}$, that is, the vector filled with ones of dimension $n_{2}$, is a left eigenvector of matrix $N$ satisfying $\mathbf{1}_{n_{2}}^{T} N=0$.

Proof. The proof can be carried out by direct computation.

Concerning matrix $H_{1 L} \in \mathbb{R}^{n_{1} \times n_{5}}$, its structure comes from (13) under the condition $H_{1 R}=0$; that is, the constraint

$$
i_{1}=-H_{1 L} i_{L}
$$

must be satisfied. Thus, the columns of $H_{1 L}$ are divided into three blocks corresponding to $r, s$, and $p$ type inductors, respectively, where the third one is zero due to the fact that sources do not belong to cutsets where $p$-inductors are involved. Hence, this matrix takes the form

$$
H_{1 L}=\left[\begin{array}{lll}
M_{r} & M_{s} & 0_{p}
\end{array}\right]
$$

where one has the following:

(i) The entries different from zero of each row of $M_{r} \epsilon$ $\mathbb{R}^{n_{1} \times n_{r}}$ stand for the connection of sources with $r$ inductors.

(ii) In concordance with the partition of matrix $H_{C L}$, which in turn comes from (C.1), the ith row of $M_{r}, i=$ $1,2, \ldots, n_{1}$, must be divided into $z$ sections leading to

$$
\beta_{i}=\left[\begin{array}{llll}
\beta_{i r_{1}} & \beta_{i r_{2}} & \cdots & \beta_{i r_{z}}
\end{array}\right]
$$

where each $\beta_{i r_{j}} \in \mathbb{R}^{1 \times r_{j}}, j=1,2, \ldots, z$, has only one entry equal to 1 if the $i$ th source is connected to the $j$ th capacitor. Otherwise, the vector is zero.

(iii) Since two sources cannot be connected to the same $r$ inductor, each column of $M_{r}$ also has only one entry different from zero.

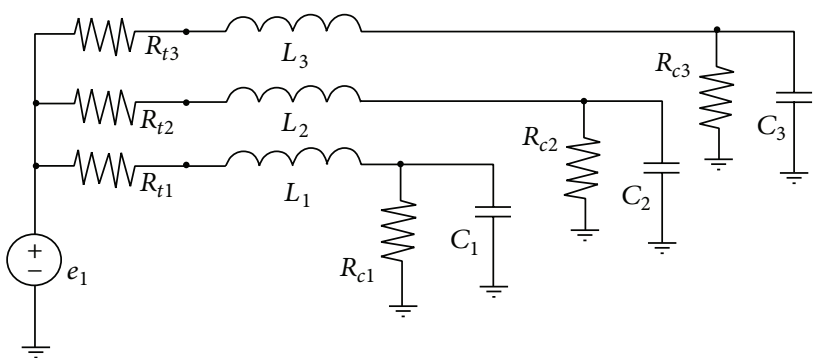

FIGURE 1: Example of radial topology.

(iv) The sum of the entries different from zero of the $i$ th row of $M_{r}$ equals $\rho_{i}$.

(v) The entries different from zero of each column of $M_{s} \in \mathbb{R}^{n_{1} \times n_{s}}$ stand for the connection of sources with other sources.

(vi) The sum of the entries different from zero of the $i$ th row of $M_{s}$ equals $\gamma_{i}$. Therefore, $\rho_{i}+\gamma_{i}=m_{i}$ of $(\mathbf{C . 3})$.

(vii) Matrix $0_{p} \in \mathbb{R}^{n_{1} \times n_{p}}$ is a zero matrix that exhibits the fact that sources cannot be related to $p$-inductors.

Due to structure (55), the following properties hold.

Property 5. The vector $\mathbf{1}_{n_{1}} \in \mathbb{R}^{n_{1}}$, that is, the vector filled with ones of dimension $n_{1}$, is a right eigenvector of matrix $M_{s}^{T}$ satisfying $M_{s}^{T} \mathbf{1}_{n_{1}}=0$.

Proof. The columns of matrix $M_{s}$ denote how a source is connected to another source. Since for each pair of sources this connection is carried out by one $s$-inductor, the current leaves one cutset and enters another, which is represented by the fact that, in each column of the matrix, only a 1 in one row and $a-1$ in another row appear. The proof is ended by direct computation.

Property 6. The following identity holds:

$$
-H_{C L}^{T} \mathbf{1}_{n_{2}}=H_{1 L}^{T} \mathbf{1}_{n_{1}}=\left[\begin{array}{c}
\mathbf{1}_{r} \\
0_{s}^{T} \\
0_{p}^{T}
\end{array}\right] \in \mathbb{R}^{n_{5}},
$$

where $\mathbf{1}_{r} \in \mathbb{R}^{n_{r}}$, with $0_{s}$ as previously defined, and $0_{p} \in \mathbb{R}^{1 \times n_{p}}$.

Proof. The proof comes as a direct consequence of the previous propositions.

The last part of this section shows that the general network presented above can be specialized to three typical circuits.

(1) Radial Network. A radial network is the simplest topology that can be found in the literature and can appear under several scenarios $[10,19,20]$. It is equipped with only one source connected to all the loads, as illustrated in Figure 1.

The main advantage of this class of circuits lies in its simplicity, which in terms of the characterization carried out 


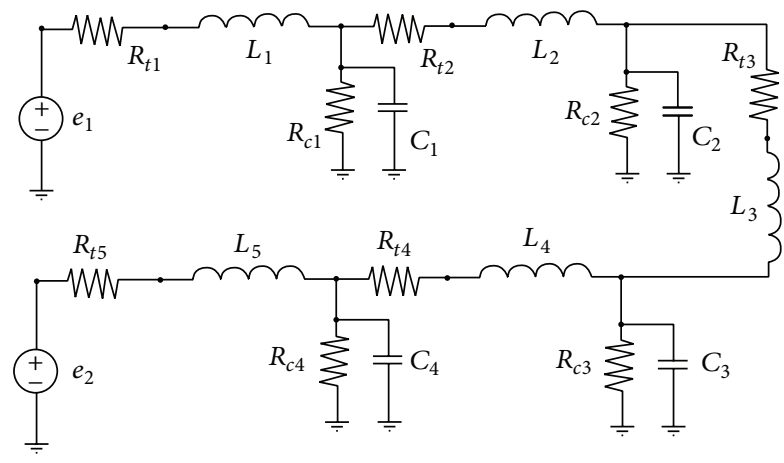

FIGURE 2: Example of ring topology.

in this paper is reflected in the fact that it is conformed only by $r$-inductors. Thus, $n_{1}=1$ and since there are as many inductors as capacitors, $n_{2}=n_{5}$.

Under these conditions, a radial network is topologically characterized by

$$
\begin{gathered}
H_{C L}=-I_{n_{2}} \\
H_{1 L}=\mathbf{1}_{n_{2}}^{T} .
\end{gathered}
$$

(2) Ring Network. This configuration looks for improving reliability by connecting each load to a second source, aiming that in case of a failure in one source the loads continue being connected to an energy supplier. One example of this kind of network is shown in Figure 2. follows:

The topological properties of this kind of circuit are as

(i) There are no $s$-inductors.

(ii) Only two sources are included $n_{1}=2$ leading to the fact that $n_{r}=2$ and that the number of capacitors that simultaneously satisfy (C.1) and (C.2) is also 2.

(iii) Due to the last item, $n_{p}=n_{2}-2$, hence, the first two row vectors of matrix $N$ are zero.

Taking into account the listed features, the matrices of a ring network take the form

$$
\begin{aligned}
-H_{C L} & =\left[\begin{array}{cc}
I_{2} & 0_{p} \\
0_{p}^{T} & N_{1}
\end{array}\right] ; \\
H_{1 L} & =\left[\begin{array}{ll}
I_{2} & 0_{p}
\end{array}\right],
\end{aligned}
$$

where in this case $0_{p} \in \mathbb{R}^{2 \times\left(n_{2}-2\right)}$ while $\mathbf{1}_{n_{2}-2}^{T} N_{1}=0$.

(3) Mesh Network. This is the most complex but at the same time the most reliable network. Its main advantage comes from the fact that every load is connected to all sources of the circuit. Actually, it can be viewed as a radial circuit with links between sources, allowing, in addition, the sources to be connected between them. A typical configuration of this class is presented in Figure 3.

Regarding the general structure introduced before, only few particularizations can be done:

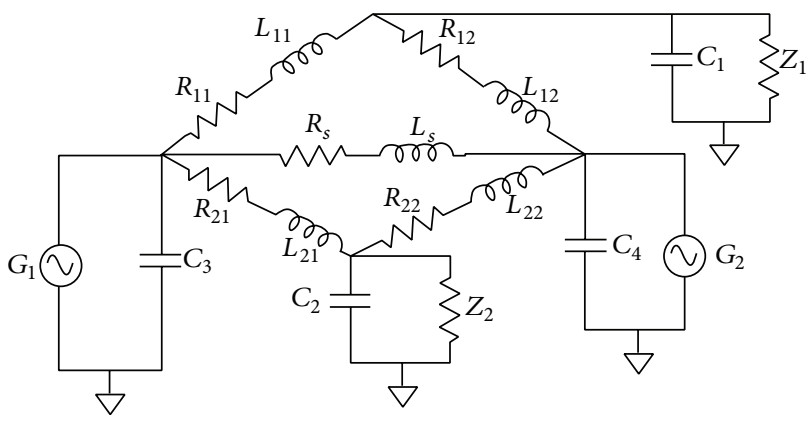

FIGURE 3: Example of mesh topology.

(i) The number of sources equals the number of capacitors; then, $n_{1}=n_{2}$.

(ii) Since all the capacitors are connected to all sources, $r_{i}=n_{1}$ for all $i \in\{1,2, \ldots, r\}$.

(iii) There are no $p$-inductors or (C.2) class capacitors.

As a consequence of the properties listed above, the matrices that topologically characterize a mesh network are

$$
H_{C L}=\left[\begin{array}{ccccc}
-\mathbf{1}_{n_{1}}^{T} & 0 & \cdots & 0 & 0_{s} \\
0 & -\mathbf{1}_{n_{1}}^{T} & \cdots & 0 & 0_{s} \\
\vdots & \vdots & \ddots & \vdots & \vdots \\
0 & 0 & \cdots & -1_{n_{1}}^{T} & 0_{s}
\end{array}\right] \in \mathbb{R}^{n_{1} \times n_{5}}
$$

where $n_{1}$ is the number of sources and $n_{s}<n_{1}$ is the number of sources connected to other sources, while

$$
H_{1 L}=\left[\begin{array}{ll}
M_{r} & M_{s}
\end{array}\right]
$$

with the particular feature that all the partitions $\beta_{i r_{j}}$ of the $i$ th row of $M_{r}$ include an element different from zero since all the sources are connected to all capacitors.

Equations (58)-(61) fully characterize the topological structure of the three typical networks. It is the authors' belief that this unified model can be used to further analyze current topologies or to develop new designs.

\section{Steady-State Characterization: Two Case Studies}

The aim of this section is to exploit the structure of the fundamental loop matrix $H$ under two different scenarios to exhibit the systematization that can be achieved to determine the dynamic behavior of a given network. Although the main objective is to point out the advantages of the approach, it is important to mention that the two case studies are closely related to situations often found in practice.

6.1. Lossless DC Network. The first case study considers a network operating under constant voltage sources, with possibly nonlinear capacitors and inductors, with linear cotree resistances and assuming that the tree resistances are equal to 
zero; that is, $R_{t}=0$. This scenario is frequently considered in practice, for example, in electrical power systems, when it is considered that the transmission lines are very long [10]. In addition, it is considered that Properties 1 and 2 hold.

Under the aforementioned conditions, model (16) reduces to

$$
\dot{x}=\left[\begin{array}{cc}
R_{c}^{-1} & -H_{C L} \\
H_{C L}^{T} & 0
\end{array}\right] \nabla_{x} H_{a}(x)+\left[\begin{array}{c}
0 \\
H_{1 L}^{T}
\end{array}\right] v_{1} .
$$

Regarding the stability properties of the network, Proposition 9 can be directly applied although in this case, due to the lossless assumption, only stability of the equilibrium point can be concluded, since

$$
\dot{H}_{0}(x)=-\left(v_{C}-v_{C}^{\star}\right)^{T} R_{c}^{-1}\left(v_{C}-v_{C}^{\star}\right) \leq 0 .
$$

However, it is easy to verify, by direct substitution of $v_{C}^{\star}$ in (62), that the maximal invariant set of the system corresponds to $i_{L}=i_{L}^{\star}$, a condition that proves that asymptotic stability is attained.

The characterization of the steady-state behavior of the circuit is defined by the equilibria of the system, which are the solutions of

$$
\begin{gathered}
-R_{c}^{-1} v_{C}^{\star}-H_{C L} i_{L}^{\star}=0 \\
H_{C L}^{T} v_{C}^{\star}+H_{1 L}^{T} v_{1}^{\star}=0 .
\end{gathered}
$$

At this point, it is clear that, for a given $v_{1}^{\star}$, the characterization can be carried out for the capacitor voltages or the inductor currents. Motivated by the stability analysis, if the capacitor voltages are chosen, it is possible to write that

$$
v_{C}^{\star}=-\left[H_{C L} H_{C L}^{T}\right]^{-1} H_{C L} H_{1 L}^{T} e_{1}^{\star}
$$

since, from Property 3, $H_{C L}$ is row full rank.

Departing from this last expression, in the following proposition, we illustrated that, exploiting the structures for $H_{C L}$ and $H_{1 L}$, it is straightforward to conclude the steadystate operation achieved by a given network. This result is illustrated for the case of a mesh circuit.

Proposition 16. Consider a mesh electrical circuit characterized by (60) and (61). Assume the following:

(i) The network is lossless; that is, $R_{t}=0$.

(ii) Propositions 6 and 9 hold.

(iii) The vector of voltage sources $v_{1}$ is composed of $n_{1}$ constant values.

Under these conditions, capacitor voltage achieves average consensus [16] in the sense that

$$
v_{C}^{\star}=\alpha \mathbf{1}_{n_{1}},
$$

with

$$
\alpha=\frac{1}{n_{1}} \sum_{m=1}^{n_{1}} v_{1 m}^{\star}
$$

being the steady-state average value of $v_{1}$.
Proof. From (60), it is directly obtained that

$$
H_{C L} H_{C L}^{T}=\left[\begin{array}{cccc}
\mathbf{1}_{n_{1}}^{T} \mathbf{1}_{n_{1}} & 0 & \cdots & 0 \\
0 & \mathbf{1}_{n_{1}}^{T} \mathbf{1}_{n_{1}} & \cdots & 0 \\
\vdots & \vdots & \ddots & \vdots \\
0 & 0 & \cdots & \mathbf{1}_{n_{1}}^{T} \mathbf{1}_{n_{1}}
\end{array}\right]
$$

leading to $\left[H_{C L} H_{C L}^{T}\right]^{-1}=\left(1 / n_{1}\right) I_{n_{1}}$, where $I_{n_{1}} \in \mathbb{R}^{n_{1} \times n_{1}}$ is the identity matrix.

On the other hand, from (61), all the partitions $\beta_{i r_{j}}^{T}$ of the columns of matrix $M_{r}^{T}$ contain an element different from zero; thus, the product

$$
-H_{C L} H_{1 L}^{T}=\left[\begin{array}{ccc}
\mathbf{1}_{n_{1}}^{T} \beta_{1 r_{1}}^{T} & \cdots & \mathbf{1}_{n_{1}}^{T} \beta_{n_{1} r_{1}}^{T} \\
\vdots & \ddots & \vdots \\
\mathbf{1}_{n_{1}}^{T} \beta_{1 r_{z}}^{T} & \cdots & \mathbf{1}_{n_{1}}^{T} \beta_{n_{1} r_{z}}^{T}
\end{array}\right]
$$

leads to the result that $-H_{C L} H_{1 L}^{T}=\mathbf{1}_{n_{1}} \mathbf{1}_{n_{1}}^{T}$, that is, an $n_{1} \times n_{1}$ matrix filled with ones.

Putting all the obtained results together in (65), it holds that

$$
v_{C}^{\star}=\frac{1}{n_{1}} \mathbf{1}_{n_{1}} \mathbf{1}_{n_{1}}^{T} v_{1}^{\star}
$$

which is equivalent to (66).

Remark 17. A direct corollary of the last proposition, which can be alternatively proved from Property 6, refers to the case when $v_{1}^{\star}=\bar{v}_{1} \mathbf{1}_{n_{1}}$, with $\bar{v}_{1} \in \mathbb{R}$. Under this condition, voltage capacitor consensus [16] is achieved in the sense that $v_{C}^{\star}=v_{1}^{\star}$.

Remark 18. It is easy to verify that for radial and ring topologies consensus on the voltages $v_{C}^{\star}$ is attained.

Remark 19. If inductive loses are included, $R_{t} \neq 0$, consensus is no longer preserved. Instead, the entries of the matrix $R_{c}^{-1}+H_{C L} R_{t}^{-1} H_{C L}^{T}$ depend on the values of the tree resistors. However, it seems that this situation allows for designing compensation techniques, that is, adding new lumped elements to the circuit, such that a prescribed behavior is accomplished.

6.2. AC Steady-State Behavior. The second approached case study consists of an electric network operating under sinusoidal voltage sources. In this case, for facility of analysis, all the passive elements are considered to be linear although, in contrast to the first case study, inductive loses are included. Hence, the circuit dynamic is described by (16) together with (23) and, for convenience, is represented as

$$
P^{-1} \dot{z}=[J-R] z+G e_{1},
$$

where $z=\left[\begin{array}{ll}v_{C}^{T} & i_{L}^{T}\end{array}\right]^{T}$ with $J, R$, and $G$ as previously defined. 
Since the steady-state behavior is now time-varying, the admissible trajectories are given as solution of

$$
P^{-1} \dot{z}^{\star}=[J-R] z^{\star}+G v_{1}^{\star}
$$

As usual [2], to carry out the analysis, it is assumed that voltage and currents are of the form

$$
f(t)=F \cos (\omega t+\phi)=\operatorname{Re}\left(\mathbb{F} e^{j \omega t}\right)
$$

with the phasor $\mathbb{F}=F e^{j \phi}$. Therefore, admissible trajectories are defined by

$$
\operatorname{Re}\left[j \omega P \mathbb{Z}^{\star} e^{j \omega t}-(J-R) \mathbb{Z}^{\star} e^{j \omega t}\right]=\operatorname{Re}(G)\left(\mathbb{V}_{1}^{\star} e^{j \omega t}\right)
$$

leading to

$$
\mathbb{Z}^{\star}=[j \omega P-(J-R)]^{-1} G \mathbb{V}_{1}^{\star}
$$

Considering that the circuit satisfies Properties 1 and 2 (i.e., identities (40) and (42) hold), the model reduces to

$$
s P-(J-R)=\left[\begin{array}{cc}
s C+R_{c}^{-1} & H_{C L} \\
-H_{C L}^{T} & s L+R_{t}
\end{array}\right],
$$

where $s=j \omega, C \in \mathbb{R}^{n_{2} \times n_{2}}$ is the capacitance matrix, and $L \in$ $\mathbb{R}^{n_{5} \times n_{5}}$ is the inductance matrix. Hence, it is obtained that

$$
\mathbb{Z}^{\star}=\left[\begin{array}{c}
\mathbb{V}_{C}^{\star} \\
\square_{L}^{\star}
\end{array}\right]=\left[\begin{array}{c}
A_{1} H_{1 L}^{T} \mathbb{V}_{1}^{\star} \\
A_{2} H_{1 L}^{T} \mathbb{V}_{1}^{\star}
\end{array}\right]
$$

with

$$
\begin{aligned}
& A_{1}=-\left[Y_{C R}+H_{C L} Y_{L R} H_{C L}^{T}\right]^{-1} H_{C L} Y_{L R} \\
& A_{2}=Y_{L R}-Y_{L R} H_{C L}^{T}\left[Y_{C R}+H_{C L} Y_{L R} H_{C L}^{T}\right]^{-1} H_{C L} Y_{L R}
\end{aligned}
$$

and diagonal admittance matrices

$$
\begin{aligned}
& Y_{C R}=s C+R_{c}^{-1} \\
& Y_{L R}=\left(s L+R_{t}\right)^{-1}=s^{-1}\left(L+s^{-1} R_{t}\right)^{-1}=s^{-1} D .
\end{aligned}
$$

In the next proposition, for illustrative purposes, the steady-state behavior of capacitor voltages is characterized assuming that the network is of the mesh type, giving continuity to the first case study. It is convenient to introduce some partitions of several matrices that facilitate the computations involved in the result.

In accordance with the results presented in Section 5, the inductance matrix takes the form

$$
L=\operatorname{diag}\left\{L_{r}, L_{s}\right\}
$$

with $L_{r} \in \mathbb{R}^{n_{r} \times n_{r}}, L_{s} \in \mathbb{R}^{n_{s} \times n_{s}}$. In addition, the former must be divided into $z$ matrices as

$$
L_{r}=\operatorname{diag}\left\{L_{r i}\right\} ; \quad i=1,2, \ldots, z,
$$

where $L_{r i} \in \mathbb{R}^{n_{1} \times n_{1}}$ since for a mesh network $n_{r}=n_{1}$.

From Properties 1 and 2, the dimension of $R_{c}$ is $n_{1} \times n_{1}$, due to the fact that $n_{1}=n_{2}$, while $R_{t} \in \mathbb{R}^{n_{5} \times n_{5}}$; that is, it equals the dimension of $L$.

Under the aforementioned partitions, matrix $D$ introduced in (80) is given by

$$
D=\operatorname{diag}\left\{D_{r}, D_{s}\right\}
$$

where $D_{r}=\operatorname{diag}\left\{D_{r i}\right\}$ with $D_{r i}=\left(L_{r i}+s^{-1} R_{t i}\right)^{-1}$, $i=1,2, \ldots, z$, and $R_{t i}$ are submatrices of $R_{t}$ of dimension corresponding to $L_{r i}$.

Concerning matrix $H_{1 L}$, whose structure is presented in (61), the submatrix $M_{r} \in \mathbb{R}^{n_{1} \times n_{r}}$ is represented as

$$
M_{r}=\left[\begin{array}{llll}
M_{r 1} & M_{r 2} & \cdots & M_{r z}
\end{array}\right]
$$

with $M_{r i} \in \mathbb{R}^{n_{1} \times r_{i}}, i=1,2, \ldots, z$, matrices composed of the vectors introduced in (56) exhibiting only one entry different from zero on each of their columns.

Proposition 20. Consider a mesh electrical circuit characterized by (60) and (61). Assume the following:

(i) Propositions 6 and 9 hold.

(ii) The vector of voltage sources $v_{1}$ is composed of $n_{1}$ sinusoidal functions.

Under these conditions, the input/output relationship between the capacitor voltage phasors $\mathbb{V}_{C}^{\star}$ and the voltage source phasors $\mathbb{V}_{1}^{\star}$ is given by

$$
\mathbb{V}_{C}^{\star}=-\mathbb{M}^{-1}\left[\begin{array}{c}
\mathbf{1}_{n_{1}}^{T} D_{r 1} M_{r 1}^{T} \\
\mathbf{1}_{n_{1}}^{T} D_{r 2} M_{r 2}^{T} \\
\vdots \\
\mathbf{1}_{n_{1}}^{T} D_{r r} M_{r z}^{T}
\end{array}\right] \mathbb{V}_{1}^{\star},
$$

where $\mathbb{M}=\left[s^{2} C+s R_{c}^{-1}+\operatorname{diag}\left\{\mathbf{1}_{n_{1}}^{T} D_{r i} \mathbf{1}_{n_{1}}\right\}\right], i=1,2, \ldots, z$.

Proof. Defining and factorizing $s=j \omega$, the first equation of (77) can be equivalently written as

$$
\mathbb{V}_{C}^{\star}=-\left[s^{2} C+s R_{c}^{-1}+H_{C L} D H_{C L}^{T}\right]^{-1} H_{C L} D H_{1 L}^{T} \mathbb{V}_{1}^{\star},
$$

where, using (60), it is straightforward to compute

$$
\begin{aligned}
& H_{C L} D H_{C L}^{T} \\
& =\left[\begin{array}{cccc}
\mathbf{1}_{n_{1}}^{T} D_{r 1} \mathbf{1}_{n_{1}} & 0 & \cdots & 0 \\
0 & \mathbf{1}_{n_{1}}^{T} D_{r 2} \mathbf{1}_{n_{1}} & \cdots & 0 \\
\vdots & \vdots & \ddots & \vdots \\
0 & 0 & \cdots & \mathbf{1}_{n_{1}}^{T} D_{r z} \mathbf{1}_{n_{1}}
\end{array}\right],
\end{aligned}
$$


while, recalling (61), it is easy to show that

$$
H_{C L} D H_{1 L}^{T}=-\left[\begin{array}{c}
\mathbf{1}_{n_{1}}^{T} D_{r 1} M_{r 1}^{T} \\
\mathbf{1}_{n_{1}}^{T} D_{r 2} M_{r 2}^{T} \\
\vdots \\
\mathbf{1}_{n_{1}}^{T} D_{r z} M_{r z}^{T}
\end{array}\right],
$$

where $M_{r}$ has been partitioned in a congruent way. (86).

The proof concludes by substituting (87) and (88) into

The usefulness of the presented result lies in the very well defined structure of (85) which allows for systematic manipulation of the capacitance, inductance, and resistance values in order to force a prescribed steady-state behavior for the capacitor voltages. For example, consider the mesh circuit of Figure 3 which has associated the matrices

$$
\begin{aligned}
& H_{1 L}=\left[\begin{array}{ccccc}
1 & 0 & 1 & 0 & 1 \\
0 & -1 & 0 & 1 & -1
\end{array}\right] \\
& H_{C L}=\left[\begin{array}{ccccc}
-1 & 1 & 0 & 0 & 0 \\
0 & 0 & -1 & -1 & 0
\end{array}\right] .
\end{aligned}
$$

In this case, the expressions for the capacitor voltages are given by

$$
\begin{aligned}
& \mathbb{V}_{C 1}^{\star}=-\frac{D_{11} \mathbb{V}_{1}^{\star}-D_{12} \mathbb{V}_{2}^{\star}}{C_{1} s^{2}+Z_{1}^{-1} s+D_{11}+D_{12}} \\
& \mathbb{V}_{C 2}^{\star}=-\frac{D_{21} \mathbb{V}_{1}^{\star}+D_{22} \mathbb{V}_{2}^{\star}}{C_{2} s^{2}+Z_{2}^{-1} s+D_{21}+D_{22}}
\end{aligned}
$$

with $D_{11}=\left(L_{11}+s^{-1} R_{11}\right), D_{12}=\left(L_{12}+s^{-1} R_{12}\right), D_{21}=$ $\left(L_{21}+s^{-1} R_{21}\right)$, and $D_{22}=\left(L_{22}+s^{-1} R_{22}\right)$. It is clear that for a given value of the voltages sources a proper choice of the circuit parameters can lead to consensus in the capacitor voltages.

Remark 21. Evidently, the idea to manipulate the capacitance, inductance, and resistance values in order to attain a given steady-state behavior is not new. Actually, in many applications, this procedure is recognized as compensation and is related to the addition of new elements parallel or series connected with the original ones. The advantage offered by the approach presented in this paper is that the compensation analysis can be carried out in a systematic way.

\section{Concluding Remarks}

In this paper a dynamic characterization of a class of electrical circuits has been presented. The approached circuit topologies are the most used in practice, namely, radial, ring, and mesh typical networks. The characterization contemplates both stability properties and steady behavior and its main feature is that it is based on the structural properties of the networks which have been obtained by using arguments from the graph theory. It has been shown that the structure of these circuits, initially conceived for reliability issues, strongly defines their dynamic behavior. The usefulness of the results reported in this paper lies in the possibility of carrying out the characterization in a very systematic way. In addition, it has been shown that these reported results offer an alternative to deal with problems like compensator location to attain a prescribed behavior.

\section{Competing Interests}

The authors declare that they have no competing interests.

\section{Acknowledgments}

Part of this work was supported by DGAPA-UNAM under Grant IN116516.

\section{References}

[1] R. K. Brayton and J. K. Moser, "A theory of nonlinear networks. I," Quarterly of Applied Mathematics, vol. 22, pp. 1-33, 1964.

[2] C. Desoer and E. Kuh, Basic Circuit Theory, McGraw-Hill, New York, NY, USA, 1969.

[3] L. Weiss and W. Mathis, "A hamiltonian formulation for complete nonlinear RLC-networks," IEEE Transactions on Circuits and Systems. I. Fundamental Theory and Applications, vol. 44, no. 9, pp. 843-846, 1997.

[4] B. M. Maschke, A. J. Van der Schaft, and P. C. Breedveld, "An intrinsic Hamiltonian formulation of the dynamics of LC-circuits," IEEE Transactions on Circuits and Systems I: Fundamental Theory and Applications, vol. 42, no. 2, pp. 73-82, 1995.

[5] D. Jeltsema and J. M. Scherpen, "A dual relation between portHamiltonian systems and the Brayton-Moser equations for nonlinear switched RLC circuits," Automatica, vol. 39, no. 6, pp. 969-979, 2003.

[6] A. J. Van der Schaft and B. M. Maschke, "Port-hamiltonian systems on graphs," SIAM Journal on Control and Optimization, vol. 51, no. 2, pp. 906-937, 2013.

[7] T. Stykel, "Balancing-related model reduction of circuit equations using topological structure," in Model Reduction for Circuit Simulation, vol. 74 of Lecture Notes in Electrical Engineering, pp. 53-83, Springer, Dordrecht, Netherlands, 2011.

[8] R. Ortega, D. Jeltsema, and J. M. Scherpen, "Power shaping: a new paradigm for stabilization of nonlinear RLC circuits," IEEE Transactions on Automatic Control, vol. 48, no. 10, pp. 1762-1767, 2003.

[9] S. Fiaz, D. Zonetti, R. Ortega, J. M. Scherpen, and A. J. van der Schaft, "A port-Hamiltonian approach to power network modeling and analysis," European Journal of Control, vol. 19, no. 6, pp. 477-485, 2013.

[10] P. Kundur, N. Balu, and M. Lauby, Power System Stability and Control, vol. 7, McGraw-Hill, New York, NY, USA, 1994.

[11] A. Van der Schaft, "Characterization and partial synthesis of the behavior of resistive circuits at their terminals," Systems \& Control Letters, vol. 59, no. 7, pp. 423-428, 2010.

[12] R. Ortega, A. van der Schaft, F. Castanos, and A. Astolfi, "Control by interconnection and standard passivity-based control 
of Port-Hamiltonian systems," IEEE Transactions on Automatic Control, vol. 53, no. 11, pp. 2527-2542, 2008.

[13] J. Bondy and S. Murty, Graph Theory with Applications, NorthHolland Publishing, 1976.

[14] B. Bollobás, Modern Graph Theory, vol. 184 of Graduate Texts in Mathematics, Springer Science \& Business Media, 1998.

[15] P. Wellstead, Introduction to Physical System Modelling, Academic Press, London, UK, 1979.

[16] H. Bai, M. Arcak, and J. Wen, Cooperative Control Design: A Systematic, Passivity-Based Approach, Springer, 2011.

[17] A. Van der Schaft, L2-Gain and Passivity in Nonlinear Control, Springer, New York, NY, USA, 1999.

[18] B. Jayawardhana, R. Ortega, E. García-Canseco, and F. Castaños, "Passivity of nonlinear incremental systems: application to PI stabilization of nonlinear RLC circuits," Systems \& Control Letters, vol. 56, no. 9-10, pp. 618-622, 2007.

[19] Westinghouse Electric Corporation, Electrical Transmission and Distribution Reference Book, Westinghouse Electric Corporation, East Pittsburgh, Pa, USA, 1965.

[20] U. Eminoglu and M. H. Hocaoglu, "A network topologybased voltage stability index for radial distribution networks," International Journal of Power and Energy Systems, vol. 29, no. 2, pp. 131-143, 2009. 


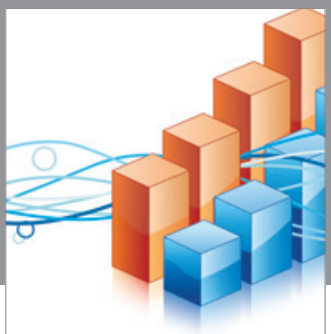

Advances in

Operations Research

vatem alat4

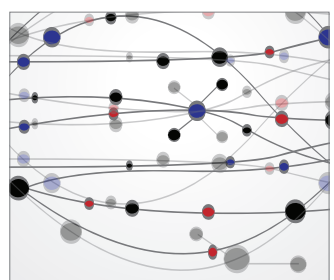

\section{The Scientific} World Journal
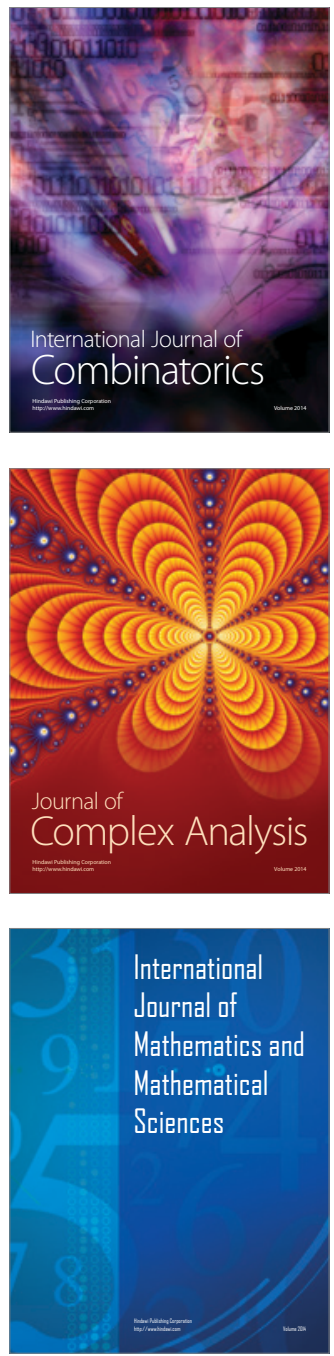
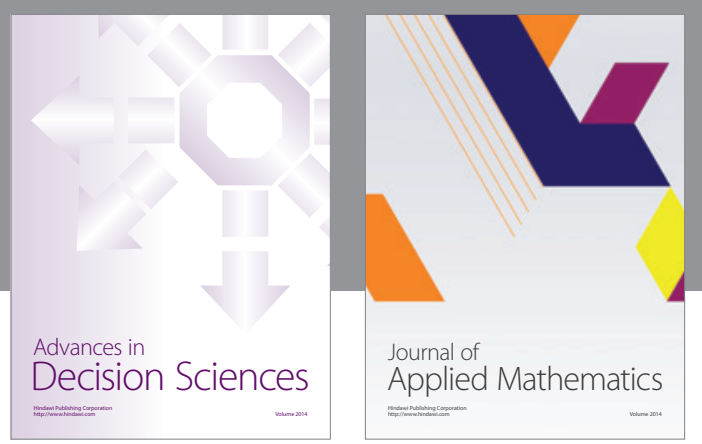

Algebra

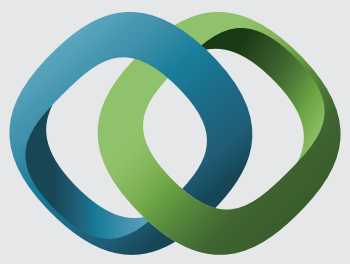

\section{Hindawi}

Submit your manuscripts at

http://www.hindawi.com
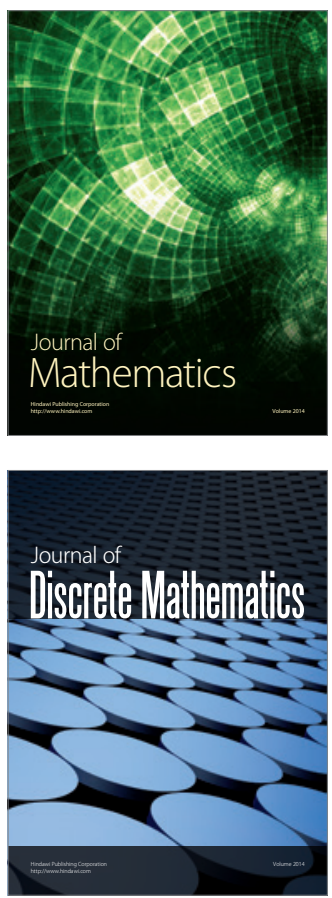

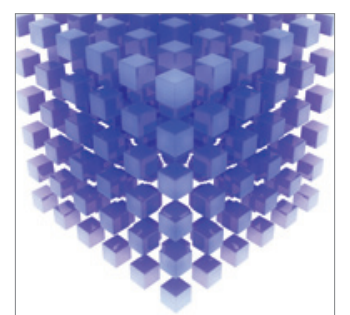

Mathematical Problems in Engineering
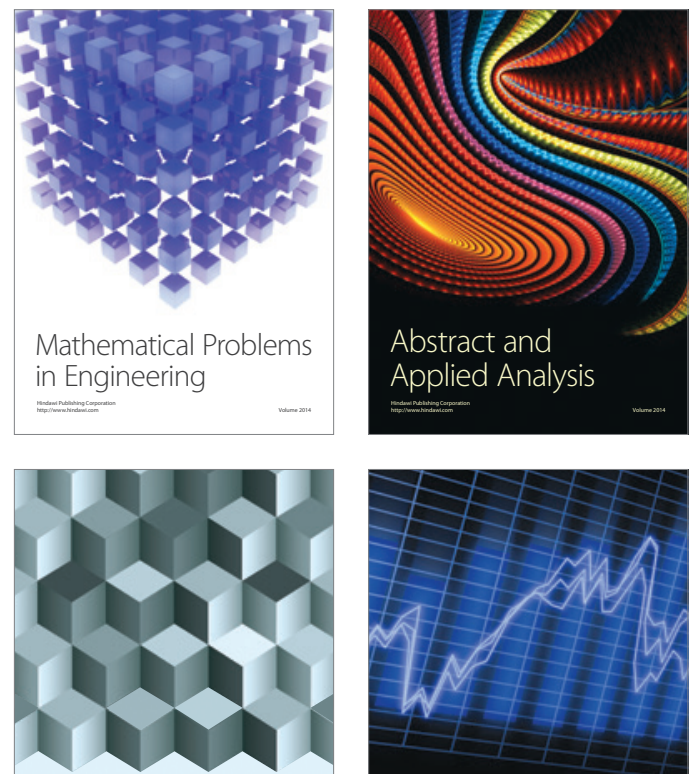

Journal of

Function Spaces

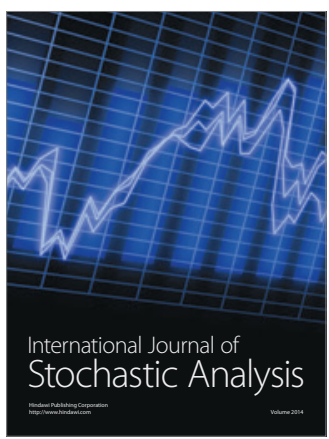

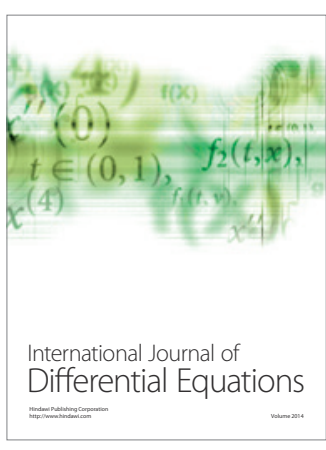
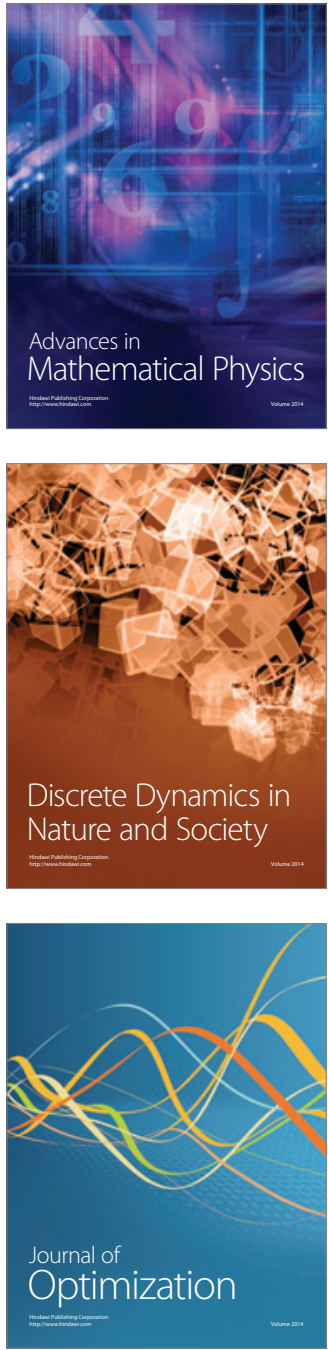